(C) 2020, The Authors. Published by FASS Inc. and Elsevier Inc. on behalf of the American Dairy Science Association ${ }^{\circledR}$. This is an open access article under the CC BY-NC-ND license (http://creativecommons.org/licenses/by-nc-nd/4.0/).

\title{
Molecular epidemiology of nonsevere clinical mastitis caused by Klebsiella pneumoniae occurring in cows on 2 Wisconsin dairy farms
}

\author{
M. J. Fuenzalida ${ }^{1}$ and P. L. Ruegg ${ }^{2 *}$ () \\ ${ }^{1}$ Department of Dairy Science, University of Wisconsin, Madison 53706 \\ ${ }^{2}$ Department of Animal Science, Michigan State University, East Lansing 48824
}

\begin{abstract}
The objective of this study was to describe diversity of Klebsiella pneumoniae isolated from milk collected at detection of nonsevere (abnormal milk or abnormal udder) clinical mastitis (CM) and during a follow-up period. Cases were detected in cows enrolled in a randomized clinical trial $(\mathrm{n}=168)$ conducted using 2 related Wisconsin dairy farms. Cases were randomly assigned to receive $2 \mathrm{~d}(\mathrm{n}=18)$ or $8 \mathrm{~d}(\mathrm{n}=18)$ of intramammary infusions with an approved product containing ceftiofur hydrochloride or assigned to a negative control group $(\mathrm{n}=17)$. Milk samples were collected from affected quarters at detection and during a 28-d followup period. Sufficient DNA was recovered from 53 of 54 Kleb. pneumoniae cultured from quarter milk samples collected at detection of the incident case. Additional Kleb. pneumoniae were recovered from milk samples collected from the same quarters at 14, 21, and $28 \mathrm{~d}$ after case detection $(\mathrm{n}=35)$, at detection of recurrent cases in the same quarter $(\mathrm{n}=14)$, and from new cases of CM ( $\mathrm{n}=3)$ occurring in enrolled quarters. All Kleb. pneumoniae were used for molecular typing by pulsedfield gel electrophoresis and $90 \%$ similarity was used to define homology. Of Kleb. pneumoniae recovered from incident cases, unique strains $(\mathrm{n}=41)$ were identified in milk samples collected from cows on farm A ( $\mathrm{n}=$ 19) and farm $B(n=22)$, whereas 12 clonal strains were identified with 8 found only in milk collected from farm A and 4 found in milk samples collected from cows on both farms. Heterogeneous strains of Kleb. pneumoniae genotypes were isolated from incident cases of CM. However, when intramammary infection persisted or when recurrence of $\mathrm{CM}$ occurred, clonal strains were isolated at 14, 21, or 28 d. Similar strains of Kleb. pneumoniae genotypes caused persistent CM.
\end{abstract}

Received August 18, 2019.

Accepted December 16, 2019.

*Corresponding author: plruegg@msu.edu
In conclusion, initial cases of CM were caused by a wide genetic diversity of Kleb. pneumoniae, but when IMI persisted, the same strain often persisted within the mammary gland up to $28 \mathrm{~d}$.

Key words: Klebsiella pneumoniae, clinical mastitis

\section{INTRODUCTION}

In many regions, environmental opportunistic pathogens have become the most common cause of clinical mastitis (CM) occurring in dairy cows (Makovec and Ruegg, 2003). Among those pathogens, environmental streptococci and gram-negative bacteria are commonly isolated (Oliveira et al., 2013). Of gram-negative pathogens, Escherichia coli and Klebsiella spp. are the most frequent causes of IMI (Oliveira et al., 2013). Clinical mastitis caused by $E$. coli is usually characterized by short-duration IMI based on an acute and effective immune response (Bannerman et al., 2004a,b; Fuenzalida and Ruegg, 2019a). In contrast, CM caused by Klebsiella spp. has been associated with longer subclinical phase IMI (Todhunter et al., 1991), unsuccessful inflammatory response, and inferior clinical outcomes (Fuenzalida and Ruegg, 2019a).

Klebsiella pneumoniae and Klebsiella oxytoca are frequently recovered from the farm environment, body sites of cow, teat-end swabs, and cases of CM (Munoz and Zadoks, 2007; Munoz et al., 2008; Zadoks et al., 2011; Rowbotham and Ruegg, 2016). In humans, Kleb. pneumoniae is commonly associated with communityacquired infections (Paterson et al., 2002). In one study, a single predominant strain of Kleb. pneumoniae was responsible for an outbreak of CM in a dairy herd in New York State (Munoz et al., 2007), but diverse strains were identified in milk collected from mastitis cows enrolled in another study conducted in the same region (Yang et al., 2019). Paulin-Curlee et al. (2007, 2008) described genetic diversity of 682 Klebsiella isolates obtained from CM detected in 7 herds and concluded that heterogeneous populations caused CM. The diversity of Klebsiella strains causing IMI 
is mostly attributed to opportunistic infection resulting from direct or indirect fecal contamination of the cow's environment (Munoz and Zadoks 2007; Munoz et al., 2007; Zadoks et al., 2011). To reduce new IMI, preventive strategies to decrease teat-end exposure to Klebsiella spp. are recommended (Paulin-Curlee et al., 2008). Based on the great diversity of Klebsiella strains, reinfection with the same strain of Klebsiella after a $\mathrm{CM}$ case has been considered unlikely (Paulin-Curlee et al., 2008). However, longitudinal studies exploring the probability of isolating the same strain after an IMI at the quarter or cow level are lacking.

Unsuccessful clearance of Klebsiella spp. from the mammary gland has been associated with the ability to evade killing by polymorphonuclear neutrophilic leukocytes (Kanevsky-Mullarky et al., 2014). Klebsiella pneumoniae can produce extended-spectrum $\beta$-lactamase that hydrolyze cephalosporins and antimicrobial resistance genes can be distributed through plasmids (Saishu et al., 2014). In our previous work describing clinical outcomes of a randomized clinical trial (Fuenzalida and Ruegg, 2019a), Kleb. pneumoniae isolates collected from nonsevere cases of CM demonstrated a bimodal distribution of phenotypic resistance to ceftiofur, indicative of possible selection for resistance. To our knowledge, no previous descriptions of genetic diversity of Kleb. pneumoniae from milk samples have been collected in a longitudinal study. The objective of this study was to describe diversity of Kleb. pneumoniae isolated from milk collected from cases of nonsevere $\mathrm{CM}$ at detection of a case, and during a defined follow-up period (FUP).

\section{MATERIALS AND METHODS}

\section{Experimental Design}

Experimental design, case enrollment criteria, data collection, and definitions have been previously described (Fuenzalida and Ruegg, 2019a). Briefly, a negatively controlled, randomized clinical trial was conducted using dairy cattle from 2 commercial Wisconsin dairy herds that had a single owner. Each farm contained more than 3,000 lactating dairy cows. In both farms (A and B), lactating cows were housed in freestall barns containing deep-bedded manure solids and were milked 3 times per day in rotary parlors.

Eligibility criteria for enrollment in the previously described randomized clinical trial were based on severity of CM and results of on-farm culture have been previously described (Fuenzalida and Ruegg, 2019a), but only cases that were confirmed as Kleb. pneumoniae in the University of Wisconsin Milk quality laboratory were used in this analysis. Nonsevere CM (appearance of abnormal milk with or without abnormal udder appearance but no generalized clinical signs) caused by gram-negative bacteria were randomly assigned to negative control (cases did not receive any antimicrobial), or 2- or 8-d intramammary treatment using a commercially available, approved product containing ceftiofur hydrochloride (Spectramast LC, Zoetis, Florham Park, NJ). Results of this trial have been previously described (Fuenzalida and Ruegg, 2019a,c) and analysis of genetic diversity of incident cases (before treatment was assigned) was performed regardless of assigned treatment.

Enrolled cases were followed for $90 \mathrm{~d}$ or until the end of lactation (FUP). During the FUP, farm workers aseptically collected milk samples from recurrent (detection of $\mathrm{CM}$ in the same enrolled quarter at least $14 \mathrm{~d}$ after case detection during the FUP) and new cases of CM (detection of CM in a different quarter at least $14 \mathrm{~d}$ after case detection during the FUP) and performed on-farm culture. Researchers (farm A) or trained farm workers (farm B) visited farms weekly to aseptically collect milk samples from enrolled quarters at $7 \mathrm{~d}$ (a single milk sample was usually collected only for SCC analysis), and duplicate milk samples at 14, 21 , and $28 \pm 3 \mathrm{~d}$ after case detection, and retrieved computerized health records and data. The d-7 milk sample was not usually cultured because cows in the 8 -d treatment group were under antibiotic therapy. One duplicate milk sample (d 14, 21, and 28) was used for confirmatory microbiological analysis at the University of Wisconsin Milk Quality laboratory and the other samples were preserved with bronopol and transported to a commercial DHIA laboratory (AgSource CRI, Verona, WI) for determination of SCC (CombiFOSS 6000, Foss Food Technology Corp., Hillerød, Denmark). For cow 5976, 2 duplicate samples were inadvertently collected at d 7 . One sample was used for determination of SCC and the other for microbiological analysis. An isolate was recovered from that sample and was used only for pulsed-field gel electrophoresis analysis (PFGE).

\section{Klebsiella pneumoniae Isolates}

Gram-negative CM cases were initially identified using on-farm culture and were enrolled in the randomized clinical trial between June and December 2016. Quarter milk samples collected at case detection and during the FUP were frozen and brought to the University of Wisconsin Milk Quality laboratory for confirmatory microbiological diagnosis following National Mastitis Council guidelines (NMC, 1999, 2017). Further description of microbiological procedures have been previously described (Fuenzalida et al., 2015; Fuenzalida and Ruegg, 
2019b). Briefly, isolates that grew on MacConkey agar were inoculated on triple sugar iron slants, motility, indole and ornithine, and sodium citrate slants. Identification of bacteria at species level was performed using API tests (API 20E, bioMerieux-Vitek Inc., Hazelwood, $\mathrm{MO})$. Pure colonies were frozen and stored at $-80^{\circ} \mathrm{C}$ until the isolate was used for PFGE.

\section{Antimicrobial Susceptibility}

In vitro antimicrobial susceptibility tests were performed for all Kleb. pneumoniae at the Wisconsin Veterinary Diagnostic Laboratory according to the Clinical and laboratory Standards Institute (CLSI, 2013). No MIC breakpoints for bovine mastitis caused by Kleb. pneumoniae are available (CLSI, 2015; VET01S), so breakpoints for $E$. coli were used. Isolates were considered resistant to ceftiofur when the MIC exceeded 4.0 $\mu \mathrm{g} / \mathrm{mL}$.

\section{Pulsed-Field Gel Electrophoresis}

The PFGE protocol was based on procedures previously described (CDC, 2017) in addition to those described in the CHEF-DR III System (Bio-Rad, Hercules, CA). Briefly, Kleb. pneumoniae isolates were cultured on Trypticase Soy Agar with 5\% defibrinated sheep blood agar plates and incubated overnight at $37^{\circ} \mathrm{C}$. Colonies from the blood agar plate were suspended in cell suspension buffer (100 $\mathrm{m} M$ Tris:100 $\mathrm{m} M$ EDTA, $\mathrm{pH}$ 8.0), and subsequently cell suspension concentrations were adjusted to absorbance of 1.00 (range of $0.8-1.0$ ) by using a spectrophotometer (610 nm wavelength). Agarose plugs were prepared by adding $10 \mu \mathrm{L}$ of proteinase $\mathrm{K}$ (20 mg/mL stock, VWR Life Science, Radnor, PA) to $200 \mu \mathrm{L}$ of the cell suspension, and gently adding $200 \mu \mathrm{L}$ of $1.2 \%$ Seakem Gold (Lonza Rockland Inc., Rockland, $\mathrm{ME}$ ) agarose at $55^{\circ} \mathrm{C}$. The mixture was transferred to plug models and allowed to solidify. Three to 4 solidified agarose plugs were transferred to $5 \mathrm{~mL}$ of proteinase $\mathrm{K}$ ( $25 \mu \mathrm{L}$ of proteinase $\mathrm{K}$ of $20 \mathrm{mg} / \mathrm{mL}$ stock solution)/ cell lysis buffer $[50 \mathrm{~m} M$ Tris (Tris-HCl, $1 M$ solution, pH 8.0, Alfa Aesar, Tewksbury, MA): $50 \mathrm{~m} M$ EDTA (EDTA, $0.5 \mathrm{M}, \mathrm{pH}$ 8.0, Promega, Madison, WI) $+1 \%$ SDS (SDS 20\% solution, Amresco Inc., Solon, OH)] and incubated in a $54-55^{\circ} \mathrm{C}$ shaker for $2 \mathrm{~h}$ with constant and vigorous agitation (150 rpm). Upon incubation, proteinase $\mathrm{K} /$ cell lysis buffer was poured off and plugs were held in tubes with a screened cap. Agarose plugs were washed twice with $10-15 \mathrm{~mL}$ sterile ultrapure water (clinical laboratory reagent water, water molecular biology grade, IBI Scientific, Dubuque, IA) and 4 times with TE buffer (10 $\mathrm{m} M$ Tris:1 $\mathrm{m} M$ EDTA, pH 8.0), both liquids preheated to 54 to $55^{\circ} \mathrm{C}$. After washing,
2.0- to 2.5-mm-wide slices from agarose plugs were cut and transferred to Eppendorf tubes containing 200- $\mu \mathrm{L}$ diluted restriction buffer $(10 \times$ restriction buffer 1:10 with clinical laboratory reagent water), then incubated in a $37^{\circ} \mathrm{C}$ water bath for 5 to $10 \mathrm{~min}$. After incubation, restriction buffer was removed and $200 \mu \mathrm{L}$ of restriction enzyme master mix containing $20 \mathrm{U} / \mu \mathrm{L}$ XbaI (New England Biolabs, Ipswich, MA) was added. Salmonella serotype Braenderup H9812 (ATCC BAA-664) ladder was used as molecular standard. Electrophoresis was carried out in $0.5 \times$ Tris-borate-EDTA buffer at $14^{\circ} \mathrm{C}$ with an initial switch time of $2.1 \mathrm{~s}$ and a final switch time of $54.1 \mathrm{~s}$ for $18 \mathrm{~h}$ at $6 \mathrm{~V} / \mathrm{cm}$. The gel was stained with ethidium bromide and destained with distilled water. Gel images were viewed and captured with Omega Lum G Gel Imaging (Aplegen, San Francisco, CA).

\section{Pulse-Field Gel Electrophoresis Analysis}

Gel images were analyzed using BioNumerics software version 7.6 (Applied Maths BVBA, Sint-Martens-Latem, Belgium). Band normalization was accomplished using the 1,135-bp Salmonella serotype Braenderup H9812 ladder (ATCC BAA-664). Band matching and isolate similarity were achieved using Dice band-based coefficient of similarity with customized tolerance and optimization of 1.0 and $1.5 \%$, respectively (Han et al., 2013). Dice coefficient $\left(S_{D}\right)$ is calculated as $S_{D}=$ $\left[2\left(\mathrm{n}_{\mathrm{xy}}\right)\right] /\left(\mathrm{n}_{\mathrm{x}}+\mathrm{n}_{\mathrm{y}}\right)$, where $\mathrm{n}_{\mathrm{xy}}$ is the number of bands shared between the 2 patterns, $n_{x}$ is the total number of bands occurring in pattern $\mathrm{X}$, and $\mathrm{n}_{\mathrm{y}}$ is the total number of bands occurring in pattern Y. Clustering was created using unweighted pair group with arithmetic averages (Han et al., 2013). A dendrogram was constructed using unweighted pair group with arithmetic averages, the relationships were identified in order of similarity, and the dendrogram was built in a stepwise manner. The percentage similarity among fingerprint patterns for each dendrogram was calculated and recorded. The cut-off to establish similarity among fingerprint patterns was 90\% based on Paulin-Curlee et al. (2007).

\section{Statistical Analysis}

Simpson's Diversity Index Calculation. Simpson's diversity index was used as a measurement of genetic diversity and was calculated using BioNumerics software version 7.6 (Applied Maths BVBA, Sint-Martens-Latem, Belgium). The formula for Simpson's diversity $(D)$ is calculated as (Paulin-Curlee et al., 2007)

$$
D=1-\frac{1}{N(N-1)} \sum_{j=1}^{S} n_{j}\left(n_{j}-1\right),
$$


where $N$ is the total number of Kleb. pneumoniae isolates from cases enrolled in the study, $S$ is the total number of strains described, and $n_{j}$ is the number belonging to the $j$ th strain.

Cophenetic Coefficient. Cophenetic correlation was used to calculate correlations between matrixes of fingerprint similarity and distance matrix (distance between 2 DNA fingerprints) using BioNumerics software version 7.6. This index indicates goodness of fit between dendrogram-derived similarities and the matrix of similarities. A value is calculated for each cluster, thus estimating the faithfulness of each subcluster of the dendrogram.

Definitions. Bacteriological cure was defined as no isolation of the same strain of Kleb. pneumoniae from milk samples collected at 14 or $21 \mathrm{~d}$ after detection of the case as compared with the strain obtained at detection. When the same strains (based on PFGE homology of $\geq 90 \%$ ) were isolated at 14,21 , or $28 \mathrm{~d}$ after detection, they were defined as persistent IMI. When cases experienced bacteriological cure at 14 and $21 \mathrm{~d}$ and later the same strain of Kleb. pneumoniae was isolated at $28 \mathrm{~d}$ after detection of the case as compared with the strain obtained at detection, these cases were considered as persistent IMI.

Description of Genetic Diversity Analysis. Genetic diversity was measured for isolates collected at case detection. Statistical analyses were conducted using SAS version 9.4 (SAS Institute, 2011). Based on similarity, isolates were classified as clonal or unique. Categorical explanatory variables were parity group (primiparous, multiparous), stage of lactation (1-30, 31-70, 71-120, or >120 d), severity score (mild or moderate), season of case detection (warm or cool), antimicrobial resistance to ceftiofur (yes or no), subclinical mastitis (SCM, DHIA SCC >150,000 cells per $\mathrm{mL}$ ) history, and previous CM (occurrence of $\mathrm{CM}$ in the same enrolled quarter within $55 \mathrm{~d}$ before case detection). Somatic cell count data were logtransformed.

Descriptive statistics for genetic diversity were performed using ANOVA of PROC GLM and chi-squared or Fisher's exact test of PROC FREQ. Univariate associations between categorical outcomes and selected risk factors were calculated using PROC LOGISTIC, chi-squared, or Fisher's exact test with PROC FREQ.

\section{RESULTS}

\section{Description of Strains Isolated at Case Detection}

Pure colonies of Kleb. pneumoniae were cultured from 54 milk samples collected at detection of 168 cases of gram-negative CM. Pulsed-field gel electrophoresis was not possible for one isolate due to DNA degradation (Figure 1). Of 53 typed isolates, 29 (55\%) were from quarter milk samples collected at farm A (Figure 2), and $24(45 \%)$ were from quarter milk samples collected at farm B (Figure 2). A total of 17, 18, and 18 typed isolates were obtained from cases of $\mathrm{CM}$ enrolled in the negative control, 2- or 8-d intramammary ceftiofur group, respectively (Table 1). Of these cases, Kleb. pneumoniae were recovered only from the original detected case of CM in 9 (53\%), $11(61 \%)$, and $14(77 \%)$ of CM enrolled in the negative control, 2- or 8-d intramammary ceftiofur group, respectively (Table 1 ).

Of 53 Kleb. pneumoniae strains cultured from quarter milk samples collected at detection, 12 were considered clonal and 41 were considered unique strains (Figure 2 ). The proportion of clonal or unique strains was not associated with stage of lactation, season of case detection, severity of CM, CM history, previous SCM, or resistance to ceftiofur $(P>0.296$; Table 2$)$. The proportion of clonal strains was greater for isolates collected from farm A $(34.5 \%)$ as compared with farm B (8.3\%; $P=0.024)$. Odds of isolating unique strains of Kleb. pneumoniae from CM cases were 5.8 (95\% CI 1.1, 29.8) times greater for isolates obtained from farm B as compared with farm A. Although far fewer isolates were collected from primiparous as compared with multiparous cows, the proportion of clonal strains had a tendency to be associated with parity $(P=0.070)$. The probability of isolating unique strains of Kleb. pneumoniae was $6.5(95 \%$ CI $0.9,44.8)$ times greater for isolates obtained from multiparous cows as compared with primiparous cows. Of isolates collected from primiparous cows, $60 \%$ (3 of 5) were clonal. In contrast, of isolates collected from multiparous cows, $18 \%$ (9 of 48) were clonal.

A total of 19 and 22 unique strains were identified in milk samples collected from cows on farm A and B, respectively (Table 2). Five distinct clonal strains were identified in quarter milk samples collected at case detection (Figure 2). Strains I $(\mathrm{n}=2)$ and V $(\mathrm{n}=2)$ were cultured from milk samples collected from cows located on both farms (Table 1), whereas strains II (n $=2)$, III $(\mathrm{n}=3)$, and IV $(\mathrm{n}=3)$ were found only in milk samples collected from cows on farm A (Figure 2).

The study had limited power to detect small differences in the proportion of clonal strains by stage of lactation, season of case detection, severity of CM, CM history, previous SCM, or resistance to ceftiofur. Cophenetic correlation was greater $(>90 \%)$ for the subcluster that had higher similarity, and as subclusters merged with other subclusters, they become less correlated (Figure 2). 


\section{Ceftiofur Susceptibility}

Of 53 Klebsiella pneumoniae isolates recovered from incident cases of CM, the MIC of $32 \%$ of Kleb. pneumoniae exceeded $\geq 4.0 \mu \mathrm{g} / \mathrm{mL}$ and (based on CLSI breakpoint for $E$. coli) were considered resistant.

The proportion of Kleb. pneumoniae considered resistant to ceftiofur was not associated with strain characteristics (clonal or unique strain; $P=0.490$; Table 2). Of clonal strains, $41 \%(\mathrm{n}=5 / 12)$ were considered resistant to ceftiofur. Of unique strains, $29 \%(\mathrm{n}=12 / 41)$ were considered resistant to ceftiofur (Table 2). Genetic diversity of Kleb. pneumoniae isolates causing incident $\mathrm{CM}$ cases was not associated with ceftiofur resistance $(P=0.227$, Table 3$)$.

\section{Description of Genetic Diversity of Strains Isolated at Detection of the Incident Case}

Genetic diversity of Kleb. pneumoniae was assessed for isolates cultured from quarter milk samples collected from incident cases at detection $(\mathrm{n}=53)$. The overall Simpson's diversity index was $91.5 \%$, indicating a wide diversity of Kleb. pneumoniae isolates cultured from quarter milk samples. Simpson's diversity index for the first, second, third, and fourth quartiles was $90.9,92.1,92.8$, and $94.7 \%$, respectively. Genetic diversity of Kleb. pneumoniae was not associated with farm, parity group, stage of lactation, season of case detection, severity of CM, CM history, previous SCM, or resistance to ceftiofur (Table $3 ; P>0.227$ ). However, we had limited power to detect the small differences we observed in Simpson's diversity index. For resistance to ceftiofur, the study had sufficient power to detect differences in means of Simpson's diversity index, but confidence intervals overlapped between Kleb. pneumoniae resistant (95\% CI 0.91-0.93) or susceptible (95\% CI 0.90-0.92) to ceftiofur.

\section{Description of Strains Isolated During Follow-up Period}

Among experimental groups, no association was observed between the number of quarters that contributed strains during the FUP $(P=0.548)$. Klebsiella pneumoniae were recovered during the FUP from incident cases of $\mathrm{CM}$ in $47 \%(8 / 17), 39 \%(7 / 18)$, and $22 \%$ $(4 / 18)$ of quarters enrolled in the negative control, 2 -d intramammary, or 8-d intramammary ceftiofur groups, respectively. The number of strains recovered during



Figure 1. Description of Klebsiella isolates obtained at case detection and follow-up period. From left to right, sufficient DNA was recovered from 53 of 54 Klebsiella pneumoniae cultured from quarter milk samples collected at detection of the incident case. Based on the results of follow-up samples at 14,21, and $28 \mathrm{~d}$, cases were categorized as bacteriological cure or persistent IMI. Clonal strains of Kleb. pneumoniae were recovered from milk samples collected from the same quarters at 14, 21, or $28 \mathrm{~d}$ after the case detection for cases experiencing persistent IMI. Bacteriological cure was defined as no isolation of the same strain of Kleb. pneumoniae from milk samples collected at 14 and $21 \mathrm{~d}$ after detection of the case as compared with the strain obtained at detection. Persistent IMI was defined as isolation of the same strain of Kleb. pneumoniae from milk samples collected at 14, 21, or $28 \mathrm{~d}$ after detection of the case as compared with the strain obtained at detection. When cases experienced bacteriological cure at 14 and $21 \mathrm{~d}$ and later the same strain of Kleb. pneumoniae was isolated at $28 \mathrm{~d}$ after detection of the case as compared with the strain obtained at detection, these cases were considered as persistent IMI. PFGE = pulsed-field gel electrophoresis; $\mathrm{CM}=$ clinical mastitis. 


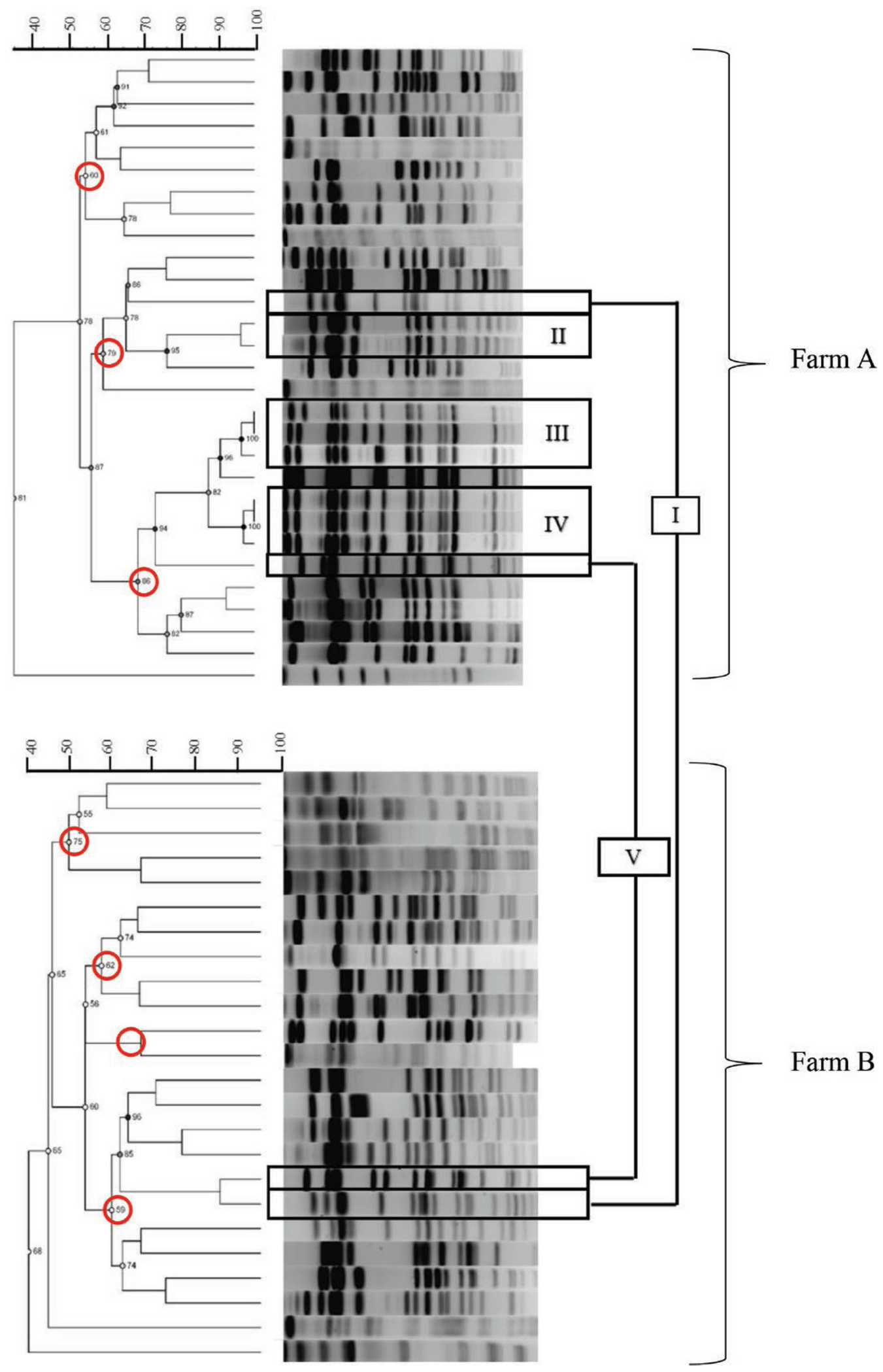

Figure 2. Clonal relationship among Klebsiella pneumoniae isolated $(\mathrm{n}=53)$ at detection of clinical mastitis cases occurring on 2 dairy herds in Wisconsin as determined by pulsed-field gel electrophoresis. Dendrogram depicts all isolates collected from farm A (top dendrogram, $\mathrm{n}=29$ ) and farm B (bottom dendrogram, $\mathrm{n}=24$ ). Roman numbers indicate distinct clonal strains. Red open circles depict the beginning of a cluster compound. Cophenetic correlation values are depicted at the beginning of cluster compounds. 
the FUP for cases allocated to the negative control (n $=8), 2$-d intramammary $(\mathrm{n}=7)$, or 8-d intramammary $(\mathrm{n}=4)$ groups were 21,22 , and 9 , respectively (Table $1)$.

Of 53 quarters from which Kleb. pneumoniae isolates were collected at case detection, follow-up samples were not available from 21 quarters either because they were not collected $(\mathrm{n}=9)$ or unusable due contamination $(\mathrm{n}=12$, Figure 1$)$. Of the remaining 32 quarters, 15 quarters experienced bacteriological cure at 14 and 21 d after case detection, but 4 Kleb. pneumoniae isolates were obtained from 3 of these quarters during the FUP (Figure 1). For 17 quarters, IMI persisted at $14(\mathrm{n}=$ 8), $21(\mathrm{n}=14)$, or $28 \mathrm{~d}(\mathrm{n}=12$; Figure 1). Strains recovered from persistently infected quarters were similar to strains recovered from incident cases but varied from strains collected from other cases. For example, a gel image indicated similarities in band patterns among Kleb. pneumoniae cultured from quarter milk samples collected at case detection, and during the FUP in 3 cows (Figure 3). For cow 5843, column 1 represents an isolate recovered at detection, columns 2 and 3 depict identical strains recovered at 21 and $28 \mathrm{~d}$ after case detection, and column 4 is a new strain recovered from a new case of CM (different from quarter enrolled in study). For cow 5976, column 5 represents an isolate recovered at case detection and column 6 is an identical strain recovered during the FUP. For cow 3469, column 7 is an isolate recovered at case detection and column 8 and 9 depict identical strains recovered during the FUP. For cow 9932, column 10 is an isolated recovered at detection and column 11 and 12 depict identical strains recovered during the FUP (Figure 3).

When strains were compared among quarter milk samples collected at detection or during the FUP, Kleb. pneumoniae strains recovered from persistent IMI were homogeneous with $\geq 90 \%$ similarity (Figure 4 ). With the exception of one cow, homogeneity was a common characteristic among all strains recovered from persistent IMI. In cow 9317, diverse strains were identified in milk samples collected at detection and d 14. Strains recovered in milk samples collected at detection from cow 4106 and cow 3677 were similar to the strain recovered in quarter milk collected $14 \mathrm{~d}$ after detection of the case occurring in cow 4654 (Figure 4). Strains recovered from cow 4654 at detection and 14 and $21 \mathrm{~d}$ during the FUP shared 90 to 100\% homology (Figure 4). The strain recovered from cow 5695 at detection was identical to the strain recovered 14 and $21 \mathrm{~d}$ in the FUP and $80 \%$ similar to the strain recovered at $28 \mathrm{~d}$ during the FUP.

Klebsiella pneumoniae strains were recovered from 10 cases that experienced a recurrent case of $\mathrm{CM}$ in the same quarter. Seven quarters experienced one recurrent

Table 1. Description of Klebsiella pneumoniae isolates recovered from quarter milk samples enrolled in a randomized clinical trial by experimental group and used for pulsed-field gel electrophoresis $(\mathrm{PFGE})^{1}$

\begin{tabular}{|c|c|c|c|c|c|c|c|c|}
\hline Experimental group & $\begin{array}{c}\text { Total } \\
\text { cases } \\
(\mathrm{n})\end{array}$ & $\begin{array}{l}\text { Isolates collected } \\
\text { only at detection } \\
\text { of CM (no.) }\end{array}$ & \multicolumn{6}{|c|}{ Isolates collected multiple times at and after detection of $\mathrm{CM}^{2}$ (no.) } \\
\hline 2-d IMM ceftiofur ${ }^{6}$ & 18 & 11 & 7 & 5 & 5 & 4 & 8 & - \\
\hline 8-d IMM ceftiofur ${ }^{7}$ & 18 & 14 & 4 & 1 & 3 & 2 & 1 & 2 \\
\hline
\end{tabular}

${ }^{1}$ Nonsevere clinical mastitis (CM) cases caused by gram-negative bacteria were enrolled in the study (Fuenzalida and Ruegg, 2019a). Of those, 54 cases were confirmed to be caused by Klebsiella pneumoniae. Fifty-three isolates were used for PFGE; it was not possible to recover DNA from 1 isolate. Isolates used for PFGE were recovered from 2 time points: at detection $(\mathrm{n}=53)$ and 14, 21, and $28 \mathrm{~d}$ after detection $(\mathrm{n}=52)$.

${ }^{2}$ Klebsiella pneumoniae isolates were continuously recovered from the same quarter at detection of CM, during weekly milk sample collection (d 14, 21, or 28), from new cases of CM, or from recurrent cases of CM.

${ }^{3}$ Quarter-level CM recurrence was detection of a new CM event at least $14 \mathrm{~d}$ after case detection during the follow-up period (or until dry off).

${ }^{4} \mathrm{~A}$ new case of $\mathrm{CM}$ was defined when workers detected a new $\mathrm{CM}$ in a different quarter at least $14 \mathrm{~d}$ after case detection during the follow-up period (or until dry off).

${ }^{5}$ Negative control group did not receive any antimicrobial treatment. Klebsiella pneumoniae were recovered from quarters of cows at detection of 17 cases of CM. Of those cases, 9 isolates were only recovered at CM detection because at 14, 21, or 28 d no isolates were recovered in the same or different quarter, or weekly quarter milk samples were missing, or unusable due to contamination.

${ }^{6}$ Quarters received a 2-d intramammary treatment using a commercially available, approved product containing ceftiofur hydrochloride. Klebsiella pneumoniae were recovered from quarters of cows at detection of 18 cases of CM. Of those cases, 11 were only recovered at CM detection because at 14,21 , or $28 \mathrm{~d}$ no isolates were recovered in the same or different quarter, or weekly quarter milk samples were missing, or unusable due to contamination.

${ }^{7}$ Quarters received an 8-d intramammary treatment using a commercially available, approved product containing ceftiofur hydrochloride. Klebsiella pneumoniae were recovered from quarters of cows at detection of 18 cases of CM. Of those cases, 14 were only recovered at CM detection because at 14,21 , or $28 \mathrm{~d}$ no isolates were recovered in the same or different quarter, or weekly quarter milk samples were missing, or unusable due to contamination. 
case of CM, 2 quarters experienced 2 recurrent cases of CM, and 1 quarter experienced 3 cases of recurrent CM. Of the 14 strains isolated from recurrent cases, 24, 36 , and $11 \%$ of the strains were recovered from cases enrolled in the negative control, 2-d intramammary, or 8-d intramammary ceftiofur groups, respectively (Table 1). Based on a $90 \%$ cut-off, all strains recovered from recurrent cases of CM were similar to the strain originally identified at detection (Figure 4).

Three strains were recovered from new cases of CM (different quarters of a previously affected cow) from 3 separate cows (4106, 5843, and 5695). Two strains were recovered from cases enrolled in the 8-d intramammary ceftiofur group and the other strain was recovered from a case enrolled in the negative control group (Table 1). One strain was recovered from cow
4106 from which Kleb. pneumoniae was not recovered during the FUP due to bacteriological cure (Figure 1). One distinct strain of Kleb. pneumoniae was recovered from milk collected from cow 5843 (Figure 3). Only one isolate recovered from a new case of CM was similar to the original isolate recovered from an incident case (cow 5695; Figure 4). A gel image compared differences among Kleb. pneumoniae strains cultured from quarter milk samples collected at detection (columns 1, 5, 7, and 10), and during the FUP (columns 2-3, 6, 8-9, and 11-12), and strains cultured from a new case of CM (column 4, Figure 4). Two of 3 strains recovered from new cases of $\mathrm{CM}$ were dissimilar to strains recovered at case detection. The strain recovered from the new CM occurring in cow 5695 was $>95 \%$ similar to the strain recovered from cow 1795 at detection. The strain recov-

Table 2. Distribution of clones $(\mathrm{n}=12)$ and unique $(\mathrm{n}=41)$ strains cultured from quarter milk samples at clinical mastitis $(\mathrm{CM})$ detection $(\mathrm{n}=53)^{1}$

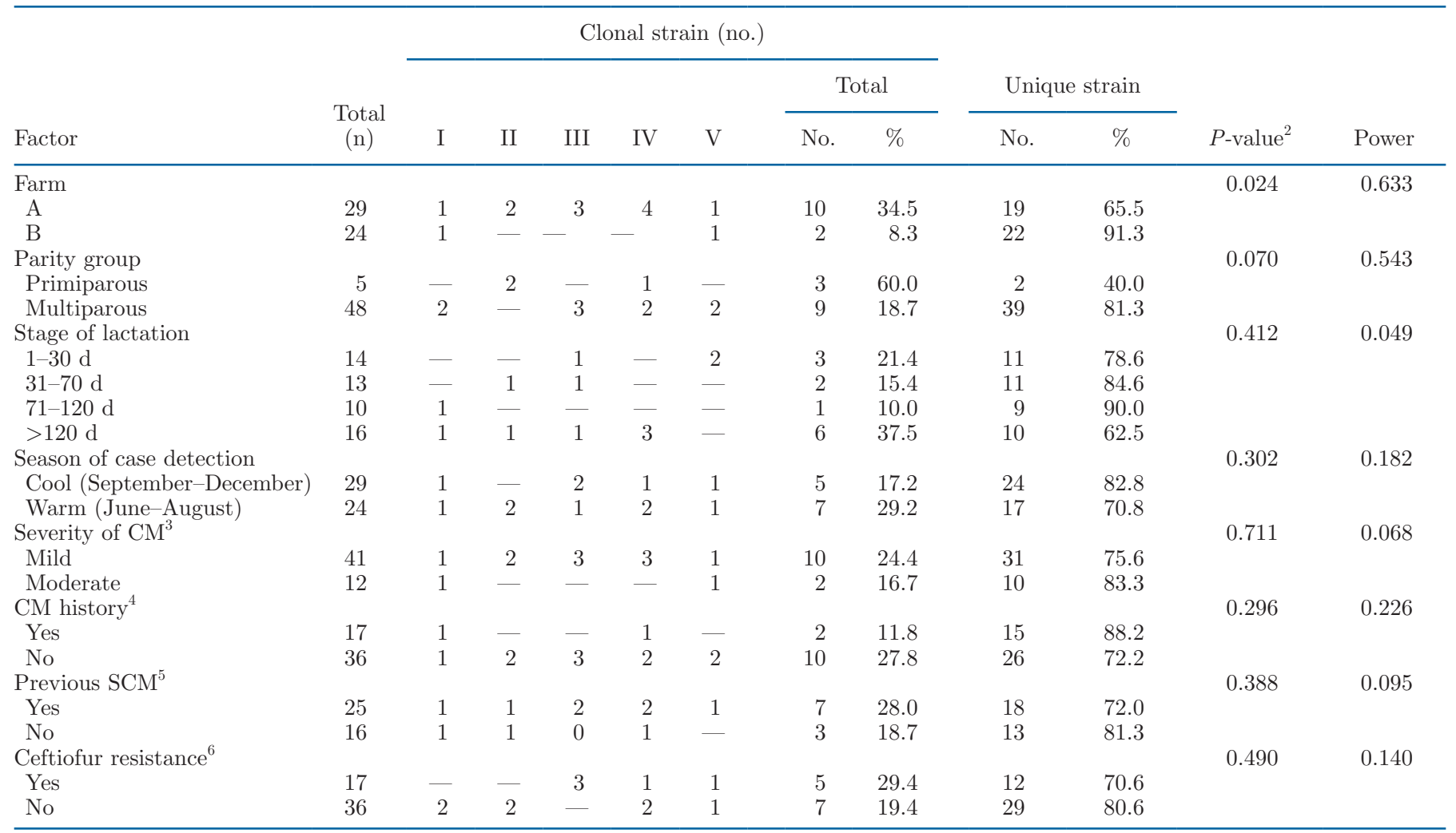

${ }^{1}$ Clonal strains were strains with more than $90 \%$ of similarity among band patterns. Unique strains were strains with less than $90 \%$ of similarity among band patterns. Percentages for clonal and unique strains were calculated by dividing the number of strains (clonal or unique) per factor by the total number of strains (clonal or unique).

${ }^{2}$ Comparisons between proportion of clones and farm or parity group or season of case detection or severity of CM or CM history or previous subclinical mastitis (SCM) or ceftiofur resistance were performed using chi-squared or Fisher's exact test.

${ }^{3}$ Severity of CM was categorized as mild (abnormal milk) or moderate (abnormal milk accompanied by inflammation of the udder).

${ }^{4}$ Previous CM history in the same enrolled quarter within $55 \mathrm{~d}$ before case detection.

${ }^{5}$ Somatic cell count greater than 150,000 cells/mL 21 to 55 d before case detection.

${ }^{6}$ Klebsiella pneumoniae isolates were considered resistant to ceftiofur based on MIC at which ceftiofur inhibited growth of bacteria $(\geq 4.0 \mu \mathrm{g} / \mathrm{mL}$ ). 
ered from new CM occurring in cow 4106 was $>90 \%$ and $100 \%$ similar to strains recovered from cow 1420 and 4654 at detection, respectively (Figure 4).

\section{DISCUSSION}

Klebsiella species are important pathogens associated with both human and animal diseases and can cause pneumonia, liver abscesses, and urinary tract infections (Paterson et al., 2002). In dairy cows, understanding the role of Klebsiella spp. in causing persistent IMI may help us to determine therapeutic interventions and improve preventive strategies. Although there are many species of Klebsiella, Kleb. pneumoniae is the most prevalent species causing mastitis and human infection (Paterson et al., 2002; Munoz et al., 2008; Zadoks et al., 2011). A previous study described great genetic diver- sity of Klebsiella spp. isolated from CM, but they did not describe associations between persistence of IMI and genetic diversity (Paulin-Curlee et al., 2008).

Sources of potential exposure to Klebsiella include water, soil, feces, bedding, and manure in cow housing areas (Zadoks et al., 2011). Researchers have described transmission of Klebsiella to cows through direct fecal exposure or through ingestion of contaminated drinking water (Zadoks et al., 2011). In our study, cows on both farms were housed in stalls containing deepbedded manure solids that are known to be a source of teat-end exposure to Klebsiella spp. (Rowbotham and Ruegg, 2016). In previous work, similar strains of Kleb. pneumoniae were found in CM cases, in bedding and in bulk tank milk, suggesting that bedding may serve as a source of bacteria that can cause bovine mastitis (Paulin-Curlee et al., 2008). Bacteria that thrive in en-

Table 3. Description of genetic diversity of Klebsiella pneumoniae isolates obtained from milk samples collected from quarters of cows at detection of clinical mastitis $(\mathrm{CM})$ on 2 related WI dairy herds $(\mathrm{n}=53)$

\begin{tabular}{|c|c|c|c|c|c|}
\hline \multirow[b]{2}{*}{ Factors } & \multirow[b]{2}{*}{$\mathrm{n}$} & \multicolumn{2}{|c|}{ Simpson's diversity } & \multirow[b]{2}{*}{$P$-value ${ }^{1}$} & \multirow[b]{2}{*}{ Power } \\
\hline & & Mean & SEM & & \\
\hline Farm & & & & 0.820 & 0.114 \\
\hline A & 29 & 0.914 & 0.004 & & \\
\hline $\mathrm{B}$ & 24 & 0.916 & 0.005 & & \\
\hline Parity group & & & & 0.915 & 0.050 \\
\hline Primiparous & 5 & 0.916 & 0.011 & & \\
\hline Multiparous & 48 & 0.915 & 0.004 & & \\
\hline Stage of lactation & & & & 0.913 & 0.052 \\
\hline $1-30 \mathrm{~d}$ & 14 & 0.916 & 0.007 & & \\
\hline $31-70 \mathrm{~d}$ & 13 & 0.918 & 0.007 & & \\
\hline $71-120 \mathrm{~d}$ & 13 & 0.915 & 0.008 & & \\
\hline$>120 \mathrm{~d}$ & 16 & 0.911 & 0.006 & & \\
\hline Season of case detection & & & & 0.593 & 0.075 \\
\hline Cool (September-December) & 29 & 0.917 & 0.005 & & \\
\hline Warm (June-August) & 24 & 0.913 & 0.005 & & \\
\hline Severity of $\mathrm{CM}^{2}$ & & & & 0.957 & 0.053 \\
\hline Mild & 41 & 0.915 & 0.004 & & \\
\hline Moderate & 12 & 0.915 & 0.007 & & \\
\hline CM history ${ }^{3}$ & & & & 0.317 & 0.209 \\
\hline Yes & 17 & 0.910 & 0.006 & & \\
\hline No & 36 & 0.917 & 0.004 & & \\
\hline Previous $\mathrm{SCM}^{4}$ & & & & 0.785 & 0.054 \\
\hline Yes & 25 & 0.916 & 0.004 & & \\
\hline No & 16 & 0.918 & 0.006 & & \\
\hline Ceftiofur resistance $^{5}$ & & & & 0.227 & 0.800 \\
\hline Yes & 17 & 0.921 & 0.006 & & \\
\hline No & 36 & 0.912 & 0.004 & & \\
\hline
\end{tabular}

${ }^{1}$ Comparisons between genetic diversity and farm or experimental group or parity group or season of case detection or severity of CM or previous CM or previous subclinical mastitis (SCM) or ceftiofur resistance were performed using ANOVA.

${ }^{2}$ Severity of CM was categorized as mild (abnormal milk) or moderate (abnormal milk accompanied by inflammation of the udder).

${ }^{3}$ Previous CM history in the same enrolled quarter within 55 d before case detection.

${ }^{4}$ Somatic cell count greater than 150,000 cells/mL 21 to 55 d before case detection.

${ }^{5}$ Klebsiella pneumoniae isolates were considered resistant to ceftiofur based on MIC at which ceftiofur inhibited growth of bacteria $(\geq 4.0 \mu \mathrm{g} / \mathrm{mL})$. 


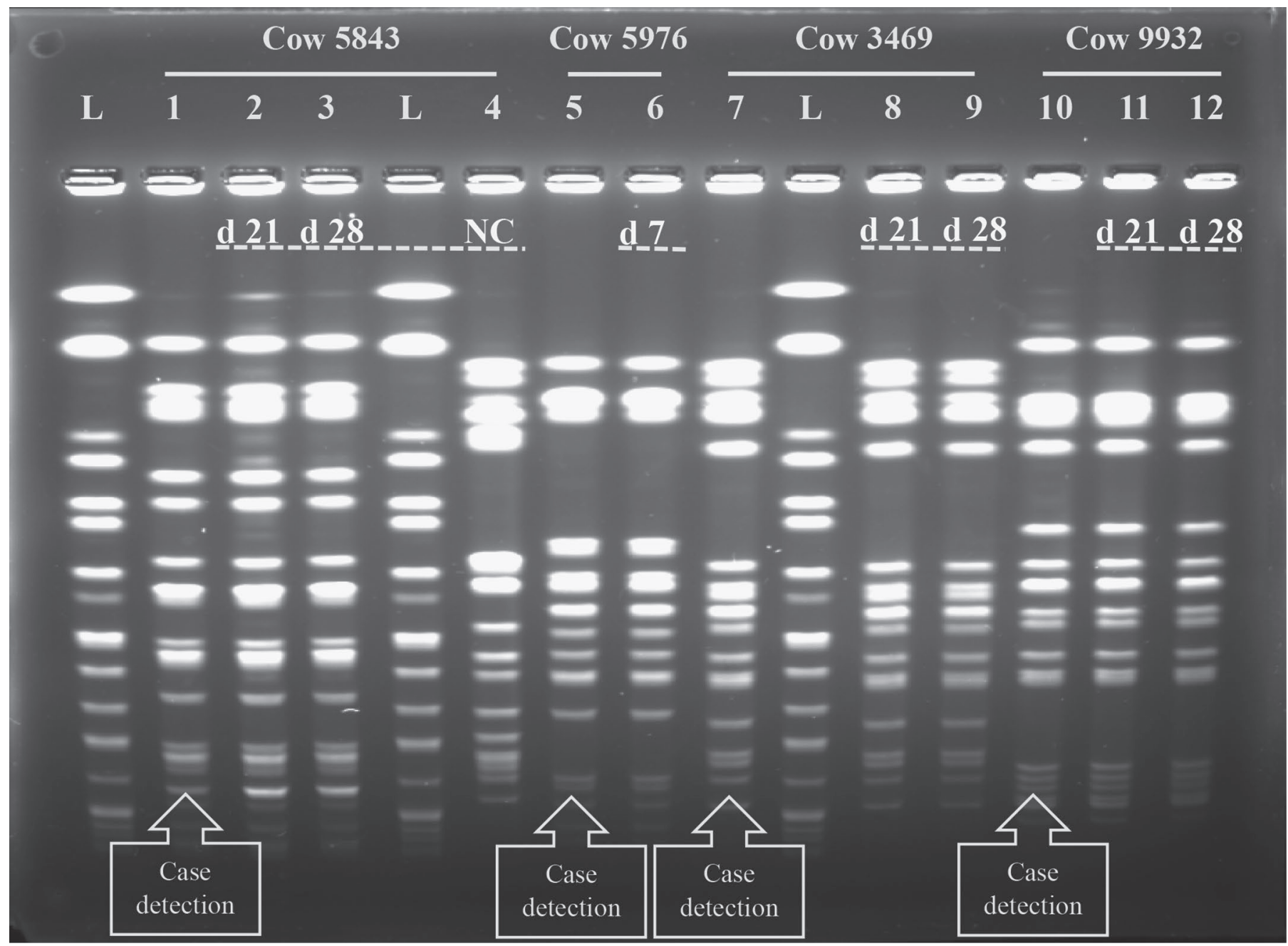

Figure 3. The DNA fingerprint patterns via XbaI digestion and pulsed-field gel electrophoresis of genomic DNA from Klebsiella pneumoniae isolates recovered from 4 cows. Columns 1, 5, 7, and 10 are isolates recovered at case detection. For cow 5843 , columns 2 and 3 are clones recovered at d 21 and 28 after case detection, whereas column 4 is a new strain. For cow 5976, column 6 is a clone obtained on d 7 after case detection. For cow 3469, columns 8 and 9 are clones recovered on d 21 and 28 after case detection. For cow 9932, columns 11 and 12 are clones recovered on d 21 and 28 after case detection. Days after case detection for every isolate recovered are depicted under the column number. Column 4 is an isolate obtained from a recurrent case of clinical mastitis in a different quarter. L represents DNA ladder. NC represents an isolate from a new case of clinical mastitis.

vironmental niches are typically diverse and cause IMI through opportunistic exposures. Our data describing great genetic diversity of the incident cases are supportive of an opportunistic exposure. However, we assessed a relatively small number of cases from just 2 farms and it is possible that more clonal isolates may have been identified if our study included more cases and more farms.

Several genotyping methods have been used to study genetic diversity among Klebsiella spp. isolates obtained from dairy herds (Paulin-Curlee et al., 2007, 2008; Munoz et al., 2007) and PFGE is one of the most used, and with greatest discriminatory power (Hansen et al., 2002; Paulin-Curlee et al., 2007, 2008; Yang et al., 2019). Paulin-Curlee et al. (2007) measured genetic diversity using 3 methods; an average of $93.6 \%$ fingerprint similarity for the same isolate across all genotyping methods was used to conservatively establish the 90\% similarity cut-off for genotype definition in that study. Yang et al. (2019) used an $80 \%$ similarity cut-off for genotype definition; no reason was given as to why that cut-off was chosen. In this study, we chose an $90 \%$ similarity cut-off. We calculated Simpson's diversity index as a measurement of genetic diversity and this index has been extensively used for this purpose by previous researchers (Munoz et al., 2007; Paulin-Curlee 


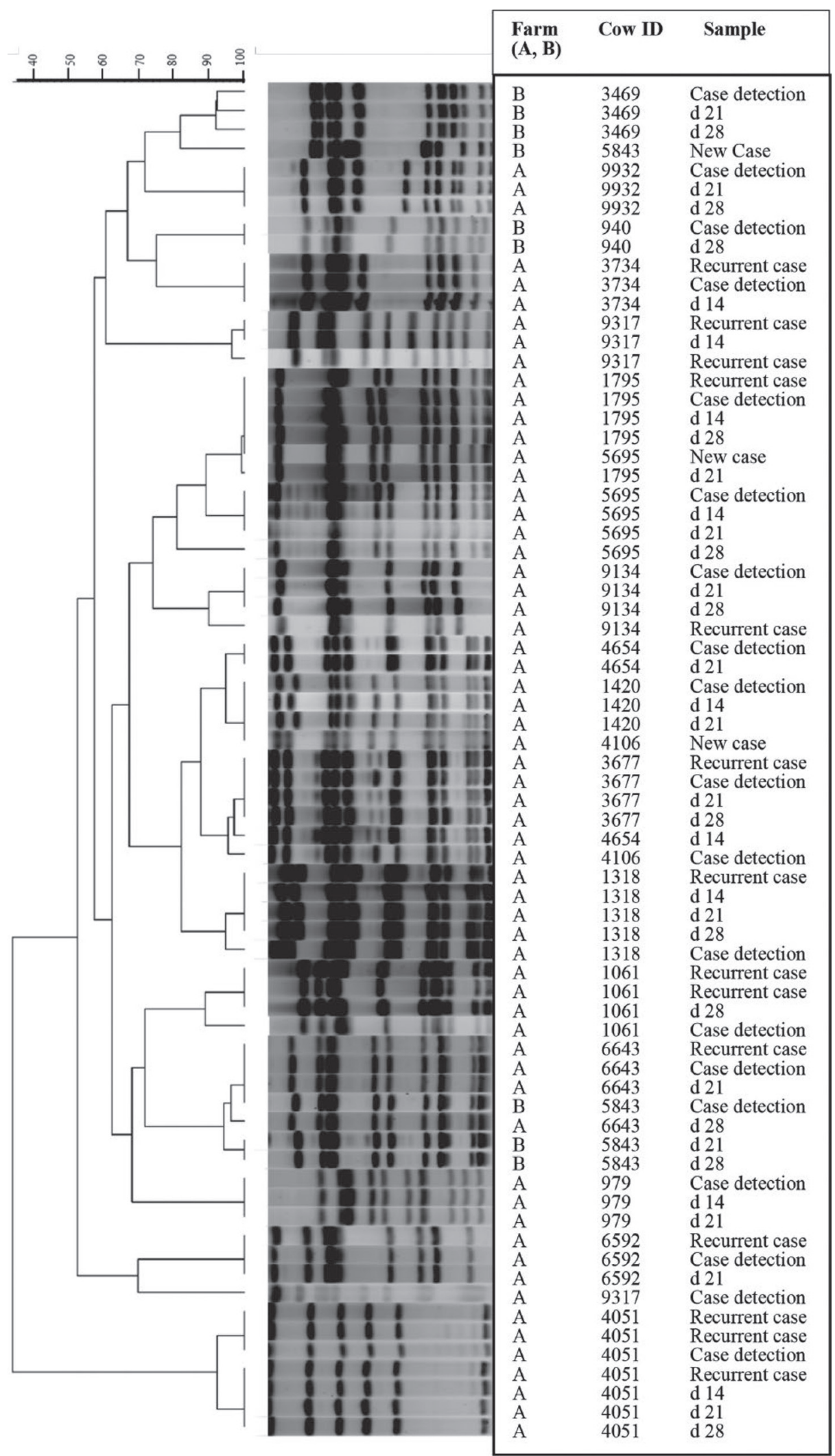

Figure 4. Clonal relationship among Klebsiella pneumoniae isolated at case detection $(\mathrm{n}=19)$, and follow-up period $(\mathrm{n}=35)$, quarterrecurrence of clinical mastitis $(\mathrm{n}=14)$, and new cases of clinical mastitis $(\mathrm{n}=3)$ as determined by pulsed-field gel electrophoresis $(\mathrm{PFGE})$. Source of the isolate is described next to the PFGE image. 
et al., 2007). As Simpson's diversity index increases, it indicates increased genetic diversity within Kleb. pneumoniae isolates (Paulin-Curlee et al., 2007).

We characterized genetic diversity of Kleb. pneumoniae isolates recovered from $\mathrm{CM}$ at detection and from milk samples collected during the FUP. These samples included both quarter-level recurrent cases of $\mathrm{CM}$ and new cases of CM. In agreement with previous reports (Paulin-Curlee et al., 2008), we observed genetic diversity in isolates collected at detection of the cases both between and within farms. We found that some clonal strains were found on both farms, but most strains were specific to a single farm, indicating that specific on-farm factors could have influenced exposure. Further research is needed to characterize associations between on-farm factors (e.g., management or environment) and genetic diversity. While few cases occurred in primiparous cows, our data indicated that parity had a tendency to be associated with the proportion of clones as we tended to observe a greater proportion of clonal strains (versus unique strains) in primiparous cows as compared with multiparous cows. This finding is especially of interest because multiparous cows are known to have poorer clinical outcomes as compared with primiparous animals (Fuenzalida and Ruegg, 2019a). The ability of cows to resist infection has been associated with parity, and younger cows typically experience less mastitis and have better clinical outcomes as compared with multiparous cows (Fuenzalida and Ruegg, 2019a,b). Our results demonstrated that ceftiofur resistance did not vary among clonal and unique Kleb. pneumoniae isolates recovered from incident cases of CM. Paulin-Curlee et al. (2008) described similar findings where the majority of genotypes were unique to herds of origin and only few genotypes were detected in more than 2 dairies. Further research with a larger number of herds and isolates is needed to study the relationship among parity, genetic diversity, and virulence factors of clonal strains causing mastitis in cows.

Using the same data set, we previously reported that bacteriological cure was increased for CM caused by Kleb. pneumoniae when a commercially available intramammary product containing ceftiofur was administered (Fuenzalida and Ruegg, 2019b). We also demonstrated that $32 \%$ of our isolates could be considered resistant to ceftiofur (using E. coli breakpoints) and failure to achieve bacteriological cure was associated with resistance (Fuenzalida and Ruegg, 2019b). Yang et al. (2019) described the emergence of pC5-like plasmids responsible for the propagation of $b l a_{\mathrm{CTM}-\mathrm{M}-1}$ and other antimicrobial resistance genes that could be associated with the use of ceftiofur for treatment of mastitis. Fuenzalida and Ruegg (2019b) described that the bacteriological cure for cases of CM caused by Kleb. pneumoniae was $21 \%$ greater in cases where cases were treated with 8-d compared with 2-d intramammary ceftiofur; however, that difference was not statistically significant. In this study, we did not observe an association between the number of strains available for PFGE and the experimental group allocation of cases enrolled in the study. Furthermore, we demonstrated that Kleb. pneumoniae strains recovered from quarter milk samples from persistent cases of CM were similar to the strain isolated from the affected quarter at detection of the case regardless to the experimental group allocation. Thus, although the clinical appearance of the milk returned to normal, it is likely that the same strain persisted within the mammary gland in a subclinical state. Likewise, when we compared strains recovered from recurrent cases of $\mathrm{CM}$ with strains recovered from the same quarter at detection, we demonstrated that the strains were also similar. In addition, we observed that strains recovered from new cases of CM were dissimilar to strains originally isolated. These results agree with Paulin-Curlee et al. (2008) who concluded that reinfection with the same strain of Klebsiella after a $\mathrm{CM}$ case was unlikely. The ability of specific strains of Kleb. pneumoniae to persist in the same quarter may be associated with specific virulence factors (KanevskyMullarky et al., 2014). Mammary tissue invasion can be explained by the pathogenicity of Kleb. pneumoniae strains causing CM, such as siderophore biosynthesis, fimbria proteins, adhesion, secretion systems (Yang et al., 2019), and capsule size (Kanevsky-Mullarky et al., 2014). It is important to mention that in some cases of CM where similar strains of Kleb. pneumoniae were recovered from quarter milk samples, strains were not always consistently recovered at 14, 21 , and $28 \mathrm{~d}$ after detection of $\mathrm{CM}$ due to no bacterial isolation, missing samples, or contamination. Based on the results of this study, experimental group allocation did not play an important role on the ability of strains of Kleb. pneumoniae to persist within the mammary gland because the same strain was recovered during the FUP. Further research is needed to identify differences in virulence factors between Klebsiella isolates that induce persistent infection as compared with strains that are rapidly eliminated from the mammary gland. Based on our data, quarters affected by Klebsiella can remain persistently infected and may serve as a point of exposure and potentially contagious transmission during milking (Munoz et al., 2007; Zadoks et al., 2011).

Among recurrent cases where Kleb. pneumoniae was recovered from the same quarter, the same strain was recovered. Although it is theoretically possible that these identical strains were the result of reinfection 
from dominant strains in the environment, the great diversity we identified in incident cases suggest that these cases were the result of persistent infection. The proportion of clonal strains varied between and within farms, but also within individual cows. Klebsiella pneumoniae is highly diverse and ubiquitous in the dairy farm environment. Many researchers have described teat cleanliness, sources of exposure, and transmission patterns that might explain the genetic diversity of Kleb. pneumoniae (Munoz et al., 2007, 2008; Zadoks et al., 2011). We have observed that different population of Klebsiella can cause CM within farms and that genetic diversity of Kleb. pneumoniae varies among farms. As every farm environment is unique, we can expect great genetic diversity that can be associated with different ways to transfer DNA among bacteria, thus accounting for some variation in the genetic diversity of this population of bacteria (Rocha, 2004).

\section{CONCLUSIONS}

A heterogeneous group of Klebsiella pneumoniae genotypes caused CM occurring in cows on 2 farms. Although many cases experienced bacteriological clearance, when Kleb. pneumoniae isolates were recovered from persistently infected cases, all strains were similar. In these 2 herds, wide genetic diversity of Kleb. pneumoniae indicates that heterogeneous population of Kleb. pneumoniae generally caused CM but persistent infections were characterized by similar strains that persisted up to $28 \mathrm{~d}$ of follow-up.

\section{ACKNOWLEDGMENTS}

We thank Terry Walker (University of Wisconsin, Madison) for helping with PFGE method and data interpretation. This study was supported by Hatch Act Formula Fund Wisconsin Grant no. WIS01799. The authors do not have any conflicts of interest.

\section{REFERENCES}

Bannerman, D. D., M. J. Paape, W. R. Hare, and J. C. Hope. 2004b. Characterization of the bovine innate immune response to intramammary infection with Klebsiella pneumoniae. J. Dairy Sci. 87:2420-2432. https://doi.org/10.3168/jds.S0022-0302(04)73365 -2 .

Bannerman, D. D., M. J. Paape, J. W. Lee, X. Zhao, J. C. Hope, and P. Rainard. 2004a. Escherichia coli and Staphylococcus aureus elicit differential innate immune responses following intramammary infection. Clin. Diagn. Lab. Immunol. 11:463-472. https://doi.org/ 10.1128/CDLI.11.3.463-472.2004.

Centers for Disease Control and Prevention (CDC). 2017. Standard Operating Procedure for PulseNet PFGE of Escherichia coli O157:H7, Escherichia coli non-O157 (STEC), Salmonella serotypes, Shigella sonnei and Shigella flexneri. Accessed Jul. 10, 2017. https://www.cdc.gov/pulsenet/pdf/ecoli-shigella-salmonella-pfge -protocol-508c.pdf.

CLSI (Clinical and Laboratory Standards Institute). 2013. Performance standards for antimicrobial disk and dilution susceptibility tests for bacteria isolated from animals; Approved standard. VET01. 4th ed. CLSI, Wayne, PA.

CLSI (Clinical and Laboratory Standards Institute). 2015. Performance standards for antimicrobial disk and dilution susceptibility tests for bacteria isolated from animals. VET01S. 3rd ed. CLSI, Wayne, PA.

Fuenzalida, M. J., P. M. Fricke, and P. L. Ruegg. 2015. The association between occurrence and severity of subclinical and clinical mastitis on pregnancies per artificial insemination at first service of Holstein cows. J. Dairy Sci. 98:3791-3805. https://doi.org/10 $.3168 /$ jds.2014-8997.

Fuenzalida, M. J., and P. L. Ruegg. 2019a. Negatively controlled, randomized clinical trial to evaluate intramammary treatment of nonsevere, gram-negative clinical mastitis. J. Dairy Sci. 102:54385457. https://doi.org/10.3168/jds.2018-16156.

Fuenzalida, M. J., and P. L. Ruegg. 2019b. Negatively controlled, randomized clinical trial to evaluate use of intramammary ceftiofur for treatment of nonsevere culture-negative clinical mastitis. J. Dairy Sci. 102:3321-3338. https://doi.org/10.3168/jds.2018-15497.

Fuenzalida, M. J., and P. L. Ruegg. 2019c. Short communication: Longitudinal study of quarter-level somatic cell responses after naturally occurring, nonsevere clinical mastitis diagnosed as culture negative, or caused by Escherichia coli or Klebsiella pneumoniae, and randomly assigned to a no-treatment group or to receive intramammary ceftiofur. J. Dairy Sci. 102:11476-11482. https://doi .org/10.3168/jds.2018-16190.

Han, H., H. Zhou, H. Li, Y. Gao, Z. Lu, K. Hu, and B. Xu. 2013. Optimization of pulse-field gel electrophoresis for subtyping of Klebsiella pneumoniae. Int. J. Environ. Res. Public Health 10:2720-2731. https://doi.org/10.3390/ijerph10072720.

Hansen, D. S., R. Skov, J. V. Benedi, V. Sperling, and H. J. Kolmos. 2002. Klebsiella typing: pulse-field gel electrophoresis (PGFE) in comparison with O:K-serotyping. Clin. Microbiol. Infect. 8:397404. https://doi.org/10.1046/j.1469-0691.2002.00411.x.

Kanevsky-Mullarky, I., A. J. Nedrow, S. Garst, W. Wark, M. Dickenson, C. S. Petersson-Wolfe, and R. N. Zadoks. 2014. Short communication: Comparison of virulence factors in Klebsiella pneumoniae strains associated with multiple or single cases of mastitis. J. Dairy Sci. 97:2213-2218. https://doi.org/10.3168/jds.2013-7140.

Makovec, J. A., and P. L. Ruegg. 2003. Antimicrobial resistance of bacteria isolated from dairy cow milk samples submitted for bacterial culture: 8,905 samples (1994-2001). JAVMA 222:1582-1589. https://doi.org/10.2460/javma.2003.222.1582.

Munoz, M. A., G. J. Bennett, C. Ahlström, H. M. Griffiths, Y. H. Schukken, and R. N. Zadoks. 2008. Cleanliness scores as indicator of Klebsiella exposure in dairy cows. J. Dairy Sci. 91:3908-3916. https://doi.org/10.3168/jds.2008-1090.

Munoz, M. A., F. L. Welcome, Y. H. Schukken, and R. N. Zadoks. 2007. Molecular epidemiology of two Klebsiella pneumoniae mastitis outbreaks on a dairy farm in New York State. J. Clin. Microbiol. 45:3964-3971. https://doi.org/10.1128/JCM.00795-07.

Munoz, M. A., and R. N. Zadoks. 2007. Short communication: Patterns of fecal shedding of Klebsiella by dairy cows. J. Dairy Sci. 90:1220-1224. https://doi.org/10.3168/jds.S0022-0302(07)71610 -7 .

National Mastitis Council. 1999. Laboratory Handbook on Bovine Mastitis. National Mastitis Council, Verona, WI.

National Mastitis Council. 2017. Laboratory Handbook on Bovine Mastitis. National Mastitis Council, Third edition.

Oliveira, L., C. Hulland, and P. L. Ruegg. 2013. Characterization of clinical mastitis occurring in cows on 50 large dairy herds in Wisconsin. J. Dairy Sci. 96:7538-7549. https://doi.org/10.3168/jds .2012-6078.

Paterson, D. L., K. L. K. Siu, and F. Chang. 2002. Klebsiella species (K. pneumoniae, K. oxytoca, K. ozaenae and K. rhinoscleromatis) in Antimicrobial Therapy and Vaccines: Microbes. Vol. I. 2nd ed. 
V. L. Yu, R. Weber, and D. Raoult, ed. Apple Trees Production, LLC.

Paulin-Curlee, G. G., R. S. Singer, S. Sreevatsan, R. Isaacson, J. Reneau, D. Foster, and R. Bey. 2007. Genetic diversity of mastitis-associated Klebsiella pneumoniae in dairy cows. J. Dairy Sci. 90:3681-3689. https://doi.org/10.3168/jds.2006-776.

Paulin-Curlee, G. G., S. Sreevatsan, R. S. Singer, R. Isaacson, J. Reneau, R. Bey, and D. Foster. 2008. Molecular subtyping of mastitis-associated Klebsiella pneumoniae isolates shows high levels of diversity within and between dairy herds. J. Dairy Sci. 91:554-563. https://doi.org/10.3168/jds.2007-0479.

Rocha, E. P. 2004. Order and disorder in bacterial outcomes. Curr. Opin. Microbiol. 7:519-527. https://doi.org/10.1016/j.mib.2004.08 .006 .

Rowbotham, R. F., and P. L. Ruegg. 2016. Bacterial counts on teat skin and in new sand, recycled sand, and recycled manure solids used as bedding in freestalls. J. Dairy Sci. 99:6594-6608. https:// doi.org/10.3168/jds.2015-10674.

Saishu, N., H. Ozaki, and T. Murase. 2014. CTX-M-Type extendedspectrum $\beta$-lactamase-producing Klebsiella pneumoniae isolated from cases of bovine mastitis in Japan. J. Vet. Med. Sci. 76:11531156. https://doi.org/10.1292/jvms.13-0120.
SAS Institute. 2011. SAS/STAT User's Guide. Version 9.4 SAS Institute Inc., Cary, NC.

Todhunter, D. A., K. L. Smith, J. S. Hogan, and P. S. Schoenberger. 1991. Gram-negative bacterial infections of the mammary gland in cows. Am. J. Vet. Res. 52:184-188.

Yang, Y., C. H. Higgins, I. Rehman, K. N. Galvao, I. L. Brito, M. L. Bicalho, J. Song, H. Wang, and R. C. Bicalho. 2019. Genomic diversity, virulence, and antimicrobial resistance of Klebsiella pneumoniae strains from cows and humans. Appl. Environ. Microbiol. 85:e02654-18. https://doi.org/10.1128/AEM.02654-18.

Zadoks, R. N. H. M. Griffiths, M. A. Munoz, C. Ahlstrom, G. J. Bennett, E. Thomas, and Y. H. Schukken. 2011. Sources of Klebsiella and Raoultella species on dairy farms: Be careful where you walk. J. Dairy Sci. 94:1045-1051. https://doi.org/10.3168/jds.2010-3603.

\section{ORCIDS}

P. L. Ruegg (1) https://orcid.org/0000-0002-7211-4512 